\title{
FORMACIÓN DE INGENIEROS EN ESPAÑA
}

\author{
Manuel Recuero López \\ Recibido el 10 de julio de 2002, aceptado el 29 de agosto de 2002
}

\begin{abstract}
RESUMEN
La formación de Ingenieros en España tiene una vieja tradición en Escuelas cuya antigüedad es superior a los 150 años. Su carácter tradicional ha sido siempre compatible con un esfuerzo permanente por adaptarse a las condiciones del contexto social, industrial e internacional de cada época. La última gran transformación se ha producido en la última década, con la ampliación del número de títulos de Ingeniería impartidos en las Universidades españolas y una importante modificación de todos los Planes de Estudio. Para cubrir de forma más adecuada la demanda de actividades profesionales que la Industria y la Administración precisa de los Ingenieros, en España existe el sistema dual basado en dos grados de Ingeniería: Los Ingenieros Técnicos o Ingenieros de primer ciclo y los Ingenieros de segundo ciclo. Los accesos de uno al otro grado están garantizados mediante un sistema de enseñanza cíclico.
\end{abstract}

Palabras claves: Enseñanza de la ingeniería, títulos y grados, especialidades

\begin{abstract}
Engineering training schools represent a tradition of more than 50 years in Spain. This spirit has always been identified by its compatibility and success for adapting to social and industrial contexts, at different times in history. The last transformation took place in the last decade, in terms of widening the number of engineering specialties granted by Spanish Universities. This transformation also took form through the upgrading of the curriculum. In order to satisfy the needs of the industry and the administration, in Spain there is a dual system that consists of two degrees in Engineering: Technical Engineers or First Cycle Engineers and Second Degree Engineers. The transferring from one program to the other is guaranteed by a cyclical teaching system.
\end{abstract}

Keywords: Engineering teaching, titles and degrees, field of expertise

\section{INTRODUCCIÓN}

Como en otros países que iniciaron su industrialización en la segunda mitad del siglo XVIII, España también conoció el surgimiento espontáneo de centros de formación en los que se preparaban a técnicos con capacidad para aplicar sus conocimientos científicos a los procesos industriales. De esta forma, la Ingeniería, que históricamente había estado vinculada a aplicaciones militares tomaba un nuevo rumbo hacia la Ingeniería civil para dar respuesta a las necesidades que generaba el nuevo desarrollo industrial.

Hasta mediados del Siglo XIX el desarrollo de las Enseñanzas Técnicas no formó parte de las Enseñanzas Oficiales y su impartición fue más fruto de las iniciativas privadas que de la Planificación del Estado. Fue el 4 de Septiembre de 1850, bajo el reinado de Isabel II, cuando se publicó el Real Decreto por el cual se incorporaban las Enseñanzas Técnicas al Régimen Oficial de los Estudios, a la vez que establecía la reglamentación según la cual se debían organizar los estudios técnicos.

El modelo según el cual se organizaron los estudios de Ingeniería en España tuvo las siguientes características:

- Las diferentes Escuelas de Ingeniería surgieron para dar respuesta a una necesidad nacional específica de disponer de determinados cuerpos de profesionales.

- Las Escuelas de Ingenierías se organizaron en centros totalmente independientes uno del otro.

\footnotetext{
${ }^{1}$ Universidad Politécnica de Madrid, Escuela Técnica Superior de Ingenieros Industriales, España, mrecuero@insia.upm.es
} 
- Su vinculación orgánica no fue a las Universidades sino a los diferentes Ministerios.

- El número de alumnos que ingresaba era muy reducido, y éstos debían superar unas fuertes pruebas de acceso.

- La formación impartida era muy generalista en la rama técnica propia de cada Ingeniería.

- Se establecieron dos grados formativos para cada una de las Ingenierías. Un primer grado, era cursada en dos años y daba lugar al título de "perito" o "ayudante" y el otro de cinco años era el que daba lugar al título de "ingeniero". Ambos se impartían en el mismo Centro y eran gestionadas por el mismo equipo directivo.

Este esquema de funcionamiento no fue modificado en lo fundamental hasta un siglo más tarde. Fue en 1957 cuando se iniciaron una serie de transformaciones profundas impulsadas por la Ley del 20 de Julio sobre "ordenación de las enseñanzas" y el posterior Decreto del 13 de Septiembre del mismo año. Las Escuelas de Ingeniería dejaron de pertenecer a los diferentes Ministerios para integrarse con su personalidad jurídica e independencia en el Ministerio de Educación y por consiguiente dentro de la Organización Universitaria General del país.

Estas Reformas también se manifestaron en los mecanismos de ingreso a las Escuelas y en la Configuración de los Planes de Estudio. Fueron anulados los duros exámenes de ingreso y se suavizó el marcado carácter generalista de los estudios de Ingeniería, incorporando ciertos niveles de especialización.

Pocos años más tarde, a partir de 1971, surgieron en España los Centros Politécnicos, algunos de las cuales se integraron en el seno de las Universidades clásicas formando lo que se denominó Universidades Mixtas y en otros casos se organizaron autónomamente formando las Universidades Politécnicas. Este fue el caso, por ejemplo de la Universidad Politécnica de Madrid cuya creación data del año 1971.

Durante todo este proceso se vino manteniendo los dos grados de titulación en cada una de las ramas de la Ingeniería y Arquitectura. Por una parte los titulados en las Escuelas Técnicas Superiores, cuya duración de los estudios fue de cinco, seis o siete años, según la época, se les identifica con el nombre de "Ingenieros" o "Arquitectos". Por otra parte los titulados de las Escuelas Universitarias de Ingeniería Técnica, cuya duración también fue modificada en distintos momentos de dos a tres o cuatro años, fueron identificados como "Ingenieros Técnicos" o "Arquitectos Técnicos". Ambas titulaciones podían ser impartidas por el mismo o por diferente Centro.

Como en los demás sistemas de enseñanza también ha existido siempre el denominado tercer ciclo conducente al título de Doctor Ingeniero, que se obtiene como una prolongación de los estudios en una Escuela Técnica Superior y que culmina con la realización y defensa de una Tesis Doctoral.

\section{INGENIEROS DE PRIMER Y SEGUNDO CICLO}

En la década de 1980, los esquemas tradicionales del funcionamiento universitario no resultaban adecuados para atender la fuerte demanda social de acceso a la Universidad ni la presión impuesta por el desarrollo industrial. Aunque los factores que pusieron en marcha una nueva transformación de la Universidad fueron múltiples, los más destacables han sido los señalados en los apartados siguientes.

\section{La Expansión de la Enseñanza Superior}

Un primer factor determinante en la transformación de la Universidad ha sido el fuerte incremento de estudiantes de enseñanza superior. Los hechos que originaron este espectacular crecimiento de la enseñanza superior fueron fundamentalmente dos:

1. La expansión demográfica de la postguerra mundial y más particularmente en España, de la postguerra civil.

2. El desarrollo económico que exigía, por una parte, la participación de obra de mano cada vez más cualificada en el mundo laboral, mientras que por otra, facilitaba la adquisición de Educación mediante la elevación del nivel de vida medio de la sociedad.

En esta situación, todas las fuerzas ideológicas, políticas y económicas, se pusieron de acuerdo en promover el desarrollo de la educación superior, aunque por motivaciones no siempre coincidentes: para unos se trataría básicamente de mejorar el rendimiento de las fuerzas productivas, mientras que otros veían la situación propicia para el progreso del nivel cultural de la sociedad, profundizando en la igualdad social y en el desarrollo de la democracia.

Como consecuencia, se produjo en España a partir de final de los años 70 un fuerte incremento de la enseñanza universitaria de la misma forma que una década antes se había producido en otros países europeos. La tabla I muestra la evolución del número de alumnos matriculados en las Universidades españolas. Es de destacar que a 
diferencia de otros países europeos, el número de alumnos no se ha estabilizado a principios de los años 90 , sino que se espera que se produzca a partir del presente curso (2000-01). La Tabla II particulariza el crecimiento del número de alumnos habido en los últimos años en las ramas técnicas de enseñanza.

Tabla I: Evolución del número de alumnos en las Universidades Españolas

\begin{tabular}{|c|c|c|}
\hline $\begin{array}{c}\text { CURSO } \\
\text { ACADÉMICO }\end{array}$ & $\begin{array}{c}\text { NÚMERO DE } \\
\text { ALUMNOS }\end{array}$ & ÍNDICE \\
\hline $1960-61$ & 167.000 & 100 \\
\hline $1970-71$ & 329.000 & 197 \\
\hline $1980-81$ & 652.000 & 390 \\
\hline $1990-91$ & 1140.000 & 682 \\
\hline $1999-00$ & 1.581 .000 & 946 \\
\hline
\end{tabular}

Tabla II: Evolución del número de alumnos en las Enseñanzas Técnicas

\begin{tabular}{|c|c|c|}
\hline $\begin{array}{c}\text { CURSO } \\
\text { ACADÉMICO }\end{array}$ & $\begin{array}{c}\text { NÚMERO DE } \\
\text { ALUMNOS }\end{array}$ & ÍNDICE \\
\hline $1994-95$ & 309.980 & 100 \\
\hline $1995-96$ & 329.019 & 106 \\
\hline $1996-97$ & 348.477 & 113 \\
\hline $1997-98$ & 358.096 & 116 \\
\hline $1998-99$ & 373.129 & 121 \\
\hline $1999-00$ & 378.346 & 123 \\
\hline
\end{tabular}

\section{El Nuevo Valor de la Educación}

La enseñanza y particularmente la enseñanza superior se ha revalorizado al ritmo que se ha producido el desarrollo tecnológico. La cualificación de los recursos humanos implicados en el sistema de producción de bienes y servicios, ha constituido uno de los elementos estratégicos en la carrera por el desarrollo económico y social, y en este interés por garantizar una buena cualificación ha destacado la formación en las Ingenierías.

La actividad profesional de los ingenieros se sitúa en el corazón de los procesos económicos de las sociedades avanzadas, pues esta profesión ha sido tradicionalmente la encargada de aplicar los conocimientos científicos al desarrollo, operación y mantenimiento de sistemas y a su optimización en términos de calidad y coste.

Consecuentemente, la enseñanza se ha convertido en sí misma en un factor de competitividad de la economía española, de la misma forma que lo es en los demás países industrializados.

\section{La Convergencia con otros Países Europeos}

En Febrero de 1976, el interés de la Comunidad Europea por la educación se concretó en la adopción de la "Resolución del Consejo y de los Ministros de Educación" sobre un programa de acción en materia de educación. En años posteriores se ha desarrollado el contexto legislativo que posibilita la construcción de una Europa sin fronteras en temas educativos.

Desde el punto de vista universitario, el gran reto planteado es el reconocimiento de currícula y de diplomas al servicio de un espacio europeo de las profesiones y de las formaciones universitarias. La plena movilidad de profesores y estudiantes exige un esfuerzo para hacer converger sistemas universitarios muy dispares y por tanto, una actitud de transformación para adaptar consensuadamente tanto los temas relativos a la organización y métodos de enseñanza como los referentes a los contenidos (conceptos, actitudes y procedimientos).

\section{LA LEY DE REFORMA UNIVERSITARIA}

El 25 de agosto de 1983 se publica la Ley de Reforma Universitaria (LRU) y con ella se abrían las puertas para una modernización de nuestra Universidad. Esta Ley promovía la Reforma de aspectos esenciales de la vida Universitaria, tales como el régimen del profesorado, la Organización Departamental o la reforma de las Enseñanzas.

Muchos analistas han apuntado que la Ley de Reforma Universitaria (LRU) ha supuesto en términos generales una mayor orientación hacia el conocido modelo formativo anglosajón, en un esfuerzo por favorecer la convergencia con otros estándares internacionales. Este hecho ha situado el actual modelo español como un modelo híbrido entre el europeo continental, con el cual le unen profundas raíces, y el modelo anglosajón.

Como consecuencia de ello, la formación de los Ingenieros en España ha experimentado en la última década algunas transformaciones que se pueden resumir en los siguientes términos:

\section{Incremento del número de títulos homologados}

La oferta de titulaciones en Ingeniería era notablemente inferior a la de otros países industrializados. Los factores que habían originado esta situación eran por una parte la dificultad de los mecanismos institucionales para la 
creación de nuevos títulos, y por otra, la caracterización legal del título como elemento definidor de ámbitos profesionales.

La LRU impulsó la creación de nuevos currícula, diversificando las titulaciones en aquellas áreas que, como es el caso de las Tecnologías de la Información, evolucionan constantemente. Las Tablas III-a y III-b muestran la relación de títulos de Ingenieros/Arquitectura que actualmente son posibles cursar en España. En la primera de ellas (Tabla III-a) se relacionan los títulos de Ingeniería de segundo ciclo o ciclo largo cuyos estudios se organizan en cinco años. En la Tabla III-b se relacionan los títulos de Ingeniería de primer ciclo cuyos estudios se organizan en tres años.

\section{Currícula diferentes para una misma titulación}

La LRU ha permitido el desarrollo de una mayor autonomía universitaria. Las Universidades han recuperado su protagonismo convirtiéndose en los centros responsables de la Concepción, Organización e impartición de las Enseñanzas.

Los Planes de Estudio conducentes a una misma titulación pueden ser substancialmente diferentes de una Universidad a otra. Asociadas a cada titulación hay unas Directrices Generales Propias que describen entre otros temas unos contenidos mínimos (llamados materias troncales) que deben impartirse obligatoriamente en todos los currícula conducentes a dicha titulación. Estas materias constituyen aproximadamente el $40 \%$ de la carga lectiva global de la titulación, por lo que cada Universidad mantiene un margen de autonomía para completar el $60 \%$ restante con materias propias de la Universidad (materias Obligatorias, Optativa o de Libre Elección).

Tabla II-a: Relación de Títulos de Ingeniería de segundo ciclo o ciclo largo y número de Universidades españolas que lo imparten (datos del curso 2000 - 01)

\begin{tabular}{|l|c|}
\hline \multicolumn{2}{|c|}{ DENOMINACIÓN DEL TÍTULO } \\
\multicolumn{1}{|c|}{ (INGENIERÍA DE CICLO LARGO) } & $\begin{array}{c}\text { N }^{\circ} \text { DE UNIVERSIDADES } \\
\text { QUE IMPARTEN EL } \\
\text { TÍTULO }\end{array}$ \\
\hline Arquitecto & 11 \\
Ingeniería Aeronáutica & 1 \\
Ingeniería Agrónomo & 14 \\
Ingeniería de Caminos, Canales y Puertos & 8 \\
Ingeniería de Minas & 3 \\
Ingeniería de Montes & 6 \\
Ingeniería de Telecomunicación & 16 \\
Ingeniería en Informática & 32 \\
Ingeniería Industrial * & 24 \\
Ingeniería Naval y Oceánico & 1 \\
Ingeniería Química & 27 \\
Ingeniería en Materiales & 7 \\
Ingeniería en Automática y Electrónica Industrial & 9 \\
Ingeniería en Electrónica & 13 \\
Ingeniería en Geodesia y Cartografía & 6 \\
Ingeniería en Organización Industrial & 11 \\
\hline
\end{tabular}

* Los estudios conducentes a esta Ingeniería son posibles realizarlos también a través de la Universidad a Distancia (UNED) que tiene más de 40 Centros asociados repartidos por toda la geografía española.

Tabla II-b: Relación de Títulos de Ingeniería de primer ciclo y número de Universidades españolas que lo imparten (datos del curso 2000 - 01) 


\begin{tabular}{|c|c|}
\hline DENOMINACIÓN DEL TÍTULO (INGENIERÍA DE PRIMER CICLO) & $\begin{array}{c}\text { NÚMERO DE } \\
\text { UNIVERSIDADES } \\
\text { QUE IMPARTEN } \\
\text { EL TÍTULO }\end{array}$ \\
\hline Arquitecto Técnico & 17 \\
\hline Ingeniería Técnica Aeronáutico & 1 \\
\hline Ingeniería Técnica Agrícola, especialidad en Explotaciones Agropecuarias & 17 \\
\hline Ingeniería Técnica Agrícola, especialidad en Hortofruticultura y Jardinería & 17 \\
\hline Ingeniería Técnica Agrícola, especialidad en Industrias Agrarias y Alimentarias & 20 \\
\hline Ingeniería Técnica Agrícola, especialidad en Mecanización y Construcciones Rurales & 6 \\
\hline Ingeniería Técnica Minera, especialidad en explotación de Minas & 9 \\
\hline Ingeniería Técnica Minera, especialidad en Instalaciones electromecánicas Mineras & 2 \\
\hline Ingeniería Técnica Minera, especialidad en Mineralurgia y Metalurgia & 4 \\
\hline Ingeniería Técnica Minera, especialidad en Recursos Energéticos, Combustibles y Explosivos & 6 \\
\hline Ingeniería Técnica Minera, especialidad en Sondeos y Prospecciones Mineras & 6 \\
\hline Ingeniería Técnica en Obras Públicas, especialidad en Construcciones Civiles & 9 \\
\hline Ingeniería Técnica en Obras Públicas, especialidad en Hidrología & 5 \\
\hline Ingeniería Técnica en Obras Públicas, especialidad en Transportes y Servicios Urbanos & 4 \\
\hline Ingeniería Técnica de Telecomunicación, especialidad en Sistemas de Telecomunicación & 8 \\
\hline Ingeniería Técnica de Telecomunicación, especialidad en Sistemas Electrónicos & 11 \\
\hline Ingeniería Técnica de Telecomunicación, especialidad en Sonido e Imagen & 7 \\
\hline Ingeniería Técnica de Telecomunicación, especialidad en Telemática & 10 \\
\hline Ingeniería Técnica en Diseño Industrial & 8 \\
\hline Ingeniería Técnica en Informática de Gestión ** & 33 \\
\hline Ingeniería Técnica en Informática de Sistemas ** & 30 \\
\hline Ingeniería Técnica en Topografía & 9 \\
\hline Ingeniería Técnica Forestal, especialidad en Explotaciones Forestales & 8 \\
\hline Ingeniería Técnica Forestal, especialidad en Industrias Forestales & 3 \\
\hline Ingeniería Técnica Industrial, especialidad en Electricidad & 22 \\
\hline Ingeniería Técnica Industrial, especialidad en Electrónica Industrial & 24 \\
\hline Ingeniería Técnica Industrial, especialidad en Mecánica & 24 \\
\hline Ingeniería Técnica Industrial, especialidad en Química Industrial & 18 \\
\hline Ingeniería Técnica Industrial, especialidad Textil & 2 \\
\hline Ingeniería Técnica Naval, especialidad en Estructuras Marinas & 3 \\
\hline Ingeniería Técnica Naval, especialidad en Propulsión y Servicios del Buque & 3 \\
\hline
\end{tabular}

** Los estudios conducentes a esta Ingeniería son posibles realizarlos también a través de la Universidad a Distancia (UNED) que tiene más de 40 Centros asociados repartidos por toda la geografía española.

En la actualidad, la mayoría de los Planes de Estudio de Ingeniería ofrecen una optatividad en torno al 20 ó

Con esto se pretende que cada Universidad pueda desarrollar un tipo de profesional más de acuerdo con su capacidad, sus medios y sobre todo con el entorno industrial en el que está ubicado.

Las Tablas III-a y III-b señalan en la columna de la derecha el número de Universidades que actualmente imparten dicha titulación. Es de señalar que en España la inmensa mayoría de las Universidades son públicas, siendo más del $90 \%$ los estudiantes de Ingeniería que están matriculados en Universidades Públicas.

\section{Mayor flexibilidad en el currículum del estudiante} $25 \%$ de la carga lectiva global, duplicando por término medio la ofrecida en los años anteriores.

La libertad del estudiante para configurar su currículum se concreta básicamente en la elección de dos tipos de asignaturas:

- Las llamadas "Optativas" mediante las cuales el estudiante puede elegir la especialización dentro de la titulación. El número de asignaturas optativas a incluir en un Plan de Estudios no está establecido por lo que constituye uno de los elementos diferenciadores entre Universidades. 
- Las llamadas "Asignaturas de Libre Elección" mediante las cuales el estudiante de Ingeniería puede completar su formación en área no específicamente técnicas: (formación humanística, técnicas de expresión oral y escrita, fundamentos y funciones de la Ingeniería, formación lingüística, ...).

Mayor énfasis en los aspectos específicos de la Ingeniería

Los Nuevos Planes de Estudio pretenden no sólo reorganizar la docencia sino principalmente innovar metodológicamente. En este sentido conviene señalar cambios cuantitativos y cualitativos. Entre los primeros se pueden señalar como principales novedades:

- Incremento de la formación tecnológica respecto a la formación científico-básica.

- Incremento de número de horas de laboratorio.

- Incorporación de las Prácticas en empresas como parte del curriculum del estudiante. Estas prácticas se desarrollan dentro de los "Programas de Cooperación Educativa" acordados entre las Universidades y Grupos de Empresas.

Como principales cambios cualitativos se pueden señalar:

- Primacía de la formación respecto a la información.

- Primacía del "saber hacer" respecto al "conocer".

- Integración de la teoría y la práctica.

- Enseñanza activa del estudiante.

- Enfoque multidisciplinar de los problemas.

Para promover un mayor protagonismo del estudiante en el proceso de enseñanza-aprendizaje, el número de horas lectivas por semana se ha reducido respecto a los Planes de Estudio anteriores a la Reforma. Actualmente el número máximo de horas lectivas por semana es de 25 de las cuales no más de 15 pueden ser de teoría.

\section{CURRÍCULUM DEL INGENIERO}

La diversidad de Ingenierías y la Autonomía de las Universidades hace difícil resumir los diferentes Planes de Estudios que actualmente se imparten a más de 350.000 estudiantes de Ingeniería/Arquitectura en nuestro país. Con los riesgos que supone la generalización de situaciones complejas, se pueden establecer algunas pautas que permitan entender mejor la formación de los ingenieros en España.

\section{Organización de los Currículos}

Las Directrices Generales Comunes para todas las enseñanzas prevén un mínimo y un máximo de veinte a treinta horas lectivas a la semana, de las cuales no más de quince corresponderán a clases teóricas. Se prevé igualmente unos límites entre 60 a 90 créditos al año, siendo un crédito el equivalente a diez horas lectivas (teóricas o prácticas). La introducción del mecanismo de créditos como unidad de cuenta de las enseñanzas permite una elaboración homogénea de Planes de Estudio y la convalidación de estudios entre enseñanzas y Universidades.

Los Planes de estudios incluyen asignaturas en las siguientes categorías:

1. Materias Troncales: Que constituyen los contenidos homogéneos mínimos correspondientes a un mismo título oficial válido en todo el territorio nacional. Suponen en torno al $40 \%$ total de la carga lectiva .

2. Materias no Troncales: Que deberán ser definidas por las Universidades al aprobar sus Planes de Estudio y podrán ser:

- Materias Obligatorias de Universidad: libremente establecidas por cada Universidad, que las incluirá en el correspondiente Plan de Estudios como obligatorias para el alumno.

- Materias Optativas de Universidad: libremente establecidas por la Universidad en el Plan de Estudios para que el alumno escoja entre las ofrecidas.

3. Materias de Libre Elección por el estudiante en orden a la flexible configuración de su currículo. Al menos el $10 \%$ del total de la carga lectiva de un Plan de Estudios quedará reservada para que el estudiante pueda cursar aquellas materias que libremente escoja entre las ofrecidas por la Universidad entre un amplio catálogo. Entre ellas se incluirán, preferentemente materias formativas de carácter general (como puede ser introducción a la filosofía, historia,...) y materias instrumentales (especialmente lenguas modernas). Con ello se fomenta la formación interdisciplinar y la flexibilidad de los currículos. 
Aunque existe cierta libertad en la organización de estudios se recomienda la organización semestral, la no existencia de asignaturas con menos de tres créditos, la limitación del número de asignaturas por semestre a seis y otro tipo de recomendaciones.

Una posible distribución de los créditos que forman un Plan de Estudios en Ingeniería puede ser tal como el mostrado en la Fig. 1.

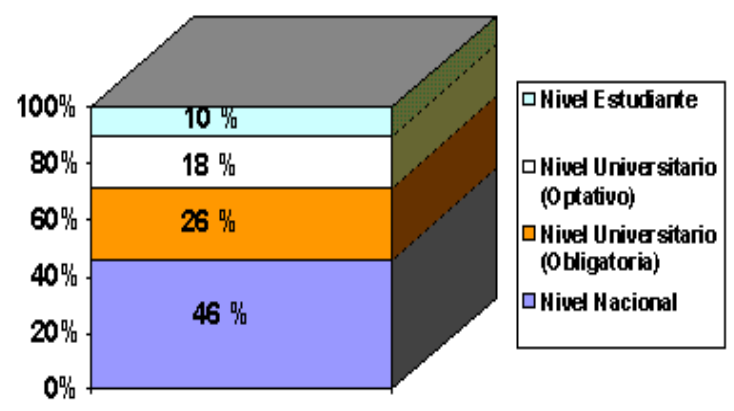

Fig. 1.- Distribución aproximada de los diferentes tipos de materias que componen un Plan de Estudios

\section{Objetivos de formación de los Ingenieros}

FEANI (Federación Europea de Asociaciones de Ingeniería) agrupa a 22 países europeos, entre ellos España, con sistemas de formación y prácticas profesionales muy diversas. Pese a ello ha sido posible consensuar los siguientes objetivos de formación del ingeniero:

Un ingeniero es una persona que ha adquirido y sabe utilizar conocimientos científicos, técnicos y cualesquiera otros necesarios que le capacitan para crear, operar y mantener sistemas eficaces, estructuras, instalaciones o procesos y para contribuir al progreso de la ingeniería mediante la investigación y el desarrollo.

Y entre otras le supone las siguientes cualidades:

a. Plena conciencia de lo que constituye la profesión $e$ ingeniero y de su responsabilidad hacia sus colegas, sus jefes, sus clientes, la sociedad y el medio ambiente.

b. Conocimiento profundo, basado en la física y las matemáticas de los fundamentos de la ingeniería de su especialidad. c. Conocimiento general de la práctica correcta de su rama de ingeniería, así como de las propiedades, comportamientos, fabricación y utilización de los materiales y de sus componentes.

d. Conocimiento de la instrumentación, de las nuevas tecnologías y capacidad de manejar información técnica y estadística.

e. Capacidad para desarrollar y utilizar modelos teóricos que simulen el comportamiento del mundo físico.

f. Capacidad para enjuiciar problemas técnicos mediante el análisis científico y la reflexión lógica.

g. Capacidad para trabajar en proyectos multidisplinares.

h. Conocimiento de las relaciones industriales y de los fundamentos de dirección empresarial en sus aspectos técnicos, financieros y humanos.

i. Facilidad para comunicarse oralmente y por escrito, y es especial para redactar informes convincentes.

j. Capacidad de encontrar, para diseños y proyectos, soluciones que combinen favorablemente la calidad con la sencillez de la producción y el mantenimiento, a un coste conveniente.

k. Apreciación positiva de los cambios tecnológicos y de la necesidad de no apoyarse solamente en prácticas establecidas sino de mantener continuamente una actitud innovadora y creativa en el ejercicio de la profesión de la ingeniería.

l. Capacidad para evaluar y sopesar factores contradictorios y heterogéneos (por ejemplo coste, calidad y tiempo) a corto plazo y para llegar a la solución ingenieril más favorable.

m. Consideración y respeto por los factores medioambientales.

n. Capacidad para movilizar recursos humanos.

o. En lo posible, dominio de otras lenguas europeas, además de la materna.

\section{Criterios de diseño currícular}

Para atender los diferentes objetivos la formación del ingeniero los Planes de Estudios se estructuran según diferentes criterios: áreas currículares, tipos de formación,... 


\section{Áreas currículares}

Tradicionalmente se establecen para Ingeniería las siguientes áreas currículares:

a. Ciencias Básicas: principalmente matemáticas y física, encargadas de proporcionar al estudiante la base y comprensión general de los fundamentos de la ingeniería y contribuir tanto a su formación como a al desarrollo de su capacidad de análisis científica.

b. Ciencias de la Ingeniería: propias de cada titulación. Tienen sus raíces en las ciencias básicas pero se distinguen de ellas por el hecho de estar orientadas hacia la aplicación creativa. Estas materias constituyen la fundamentación teórica de la ingeniería $y$ deben proporcionar un conocimiento profundo que pueda ser de utilidad durante un largo tiempo a pesar de la rápida evolución de la tecnología.

c. Diseño en Ingeniería: en donde se incluyen materias orientadas a desarrollar aplicaciones prácticas y metodologías orientadas al diseño y práctica profesional. Un especial énfasis hay que hacer en el Proyecto Fin de Carrera que los alumnos deben realizar antes de obtener el título.

d. Finalmente deben ser consideradas disciplinas que proporcionan conocimiento en Economía y Gestión Industrial, así como otras materias complementarias que aporten al alumno competencias, valores y actitudes, tales como Ciencias Sociales, Humanidades y otras disciplinas que ayuden al ingeniero a entender la cultura, la sociedad y otros aspectos de su contexto social.
Una posible distribución de estas áreas currículares se muestra en la Fig. 2. Dicha distribución debe ser interpretada como orientativa dado que la autonomía de cada Universidad permite diferentes orientaciones.

\section{Tipos de Formación}

Los Planes de Estudios permiten obtener créditos a través de tres tipos de enseñanzas/actividades: Formación teórica, formación Práctica y Prácticas en Empresas ( o trabajos académicamente dirigidos).

La formación práctica, entendiendo esta como práctica de laboratorio, constituye en la gran mayoría de los Planes de Ingeniería más del $25 \%$ de la carga lectiva global, lo cual exige fuertes inversiones en infraestructura. Este hecho obliga a los Centros y Departamentos a buscar fuentes de financiación complementarias a través de Proyectos y acuerdos con la Industria.

Obviamente la formación práctica de Laboratorio no está distribuida homogéneamente a lo argo del Plan de Estudios sino que está concentrada fundamentalmente en los últimos años de la carrera.

Otro aspecto importante son las Prácticas en empresas. El Real Decreto 1845/1994 establece que las estancias en empresa se desarrollen a través de programas de cooperación educativa y que el estudiante que desee participar en él debe haber superado el 50 por 100 de los créditos necesarios para obtener el título universitario.

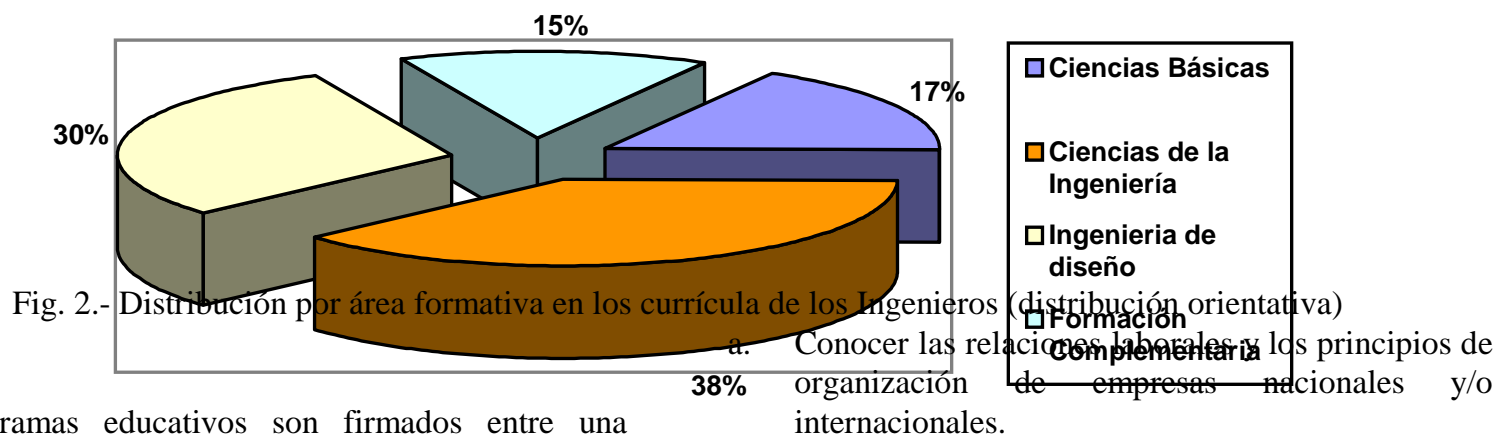

Los programas educativos son firmados entre una empresa y la Universidad y con carácter general se pueden establecer los siguientes objetivos: b. Comprender la importancia del trabajo en equipo, así como de la comunicación y de las relaciones interpersonales en el ámbito empresarial. 
c. Estimular su curiosidad intelectual y provocar una actitud más positiva hacia el aprendizaje en base a la comprobación experimental de la utilidad práctica de los conocimientos.

d. Favorecer el desarrollo y madurez personal en base a la asunción de responsabilidades y a la convivencia en una organización estructurada de forma diferenciada a la Universidad.

Estos objetivos generales deberían ser determinantes a la hora de evaluar la acreditación de la "estancia en la empresa" con independencia del tipo, actividad o titularidad jurídica de la organización laboral en la que hiciera las prácticas. Complementariamente se podrían establecer otros objetivos más específicos de acuerdo con el lugar y puesto de trabajo. Estos otros objetivos serían del tipo:

a. Utilizar equipamiento más avanzado que el disponible en la Universidad.

b. Aprender una técnica concreta.

c. Trabajar en un proyecto.

El tiempo de trabajo del estudiante en una empresa es acreditado en la relación de 30 horas de trabajo equivalente a 1 crédito siempre que el tutor académico certifique el buen aprovechamiento y la consecución de los objetivos propuestos.

\section{Los dos grados de Formación de Ingenieros: Ingeniería de Concepción e Ingeniería de Producción}

En España, como en otros países existen dos grados de formación en las diferentes Ingenierías: Ingeniero de primer ciclo o Ingeniero Técnico e Ingeniero de segundo ciclo o Ingeniero (superior). Las diferencias y similitudes entre ambos grados no siempre han sido definidos con claridad ni tampoco existen situaciones claras de referencia en otros países en los que se pueda decir que es una situación idéntica a la española. Sin embargo sí mantiene claras semejanzas con los modelos de otros países europeos y concretamente con el centro europeo, representado fundamentalmente por Alemania.

Tal vez la principal diferencia en ambas titulaciones es la orientación hacia diferentes actividades profesionales.

Para ilustrar esta idea se pueden clasificar esquemáticamente las actividades profesionales de los Ingenieros en base a la siguiente escala:

1. Investigación Básica

2. Investigación y Desarrollo

3. Proyectos: Ingeniería de Proyectos, Diseño, Estudios
4. Gestión y Administración: Dirección de Proyectos Gestión de operaciones, Sistemas de información

5. Producción: Control de procesos, Control de calidad

6. Marketing y Comercialización: Dirección comercial, Comunicación, Servicio post-venta

Esta clasificación se ha ordenado en función de los tipos de conocimientos y destrezas que el Ingeniero debe aplicar en su ejercicio profesional. Los primeros niveles se identifican con una formación eminentemente teórica, con énfasis en los principios científicos y tecnológicos y por su capacidad para analizar los sistemas y procesos matemática y físicamente. Por el contrario los últimos niveles se asocian con una formación hacia las posibilidades de la tecnología, el conocimiento de los desarrollos y aplicaciones prácticas, y los productos comerciales.

Resumiendo más aún las actividades anteriores se podrían definir dos perfiles de Ingeniería:

La Ingeniería de Concepción se identificaría con la Ingeniería de ciclo largo (o segundo ciclo) y estaría mas orientada a cubrir aquellas funciones relacionadas con actividades de Investigación, Desarrollo de Proyectos, Elaboración de Estudios.

Por su parte, la Ingeniería de Producción se identificaría con la actual Ingeniería Técnica (o de primer ciclo) y tendría una orientación profesional hacia actividades relacionadas con la Producción, Control de calidad, Desarrollo de aplicaciones, Mantenimiento.

Las investigaciones realizadas sobre las funciones desempeñadas en el ejercicio profesional por los actuales Ingenieros e Ingenieros Técnicos en España avalan este planteamiento. A modo de ejemplo se pueden citar los resultados obtenidos de sendas investigaciones llevadas a cabo sobre el colectivo de Ingenieros en el área de las Telecomunicaciones cuyo número de titulados se sitúa en torno a los 20.000. Los resultados son muy coincidentes con los llevados a cabo por las principales grandes empresas en Alemania (En este país existen también los dos tipos de ingeniería que se estudian en las Universidades y Fachohschulen) y el resumen se muestra en la Fig. 3.

La diferente orientación profesional en los dos grados de la Ingeniería están lógicamente asociados a dos perfile currículares diferentes. De todas formas, conviene señalar que aunque aquí se haga referencia a los aspectos diferenciales, hay que recordar que ambos grados de Ingeniería comparten muchos objetivos y por tanto sus enseñanzas también comparten enfoques comunes. 
El medio utilizado para expresar gráficamente el perfil de ambos grados de Ingeniería se ha tomado una vez más de los estudios y propuestas que se han realizado respecto a la Ingeniería de Telecomunicación. Para los dos grados de esta titulación se han elaborado propuestas del perfil académico utilizando el diagrama SV. Dicho diagrama es un hiperespacio en el que se refleja el nivel de actuación sobre el Procesamiento de los Contenidos y por tanto es un indicador del tipo de Ingenieros que se están formando.

\section{Teoría, Ciencia}

Investigación Básica

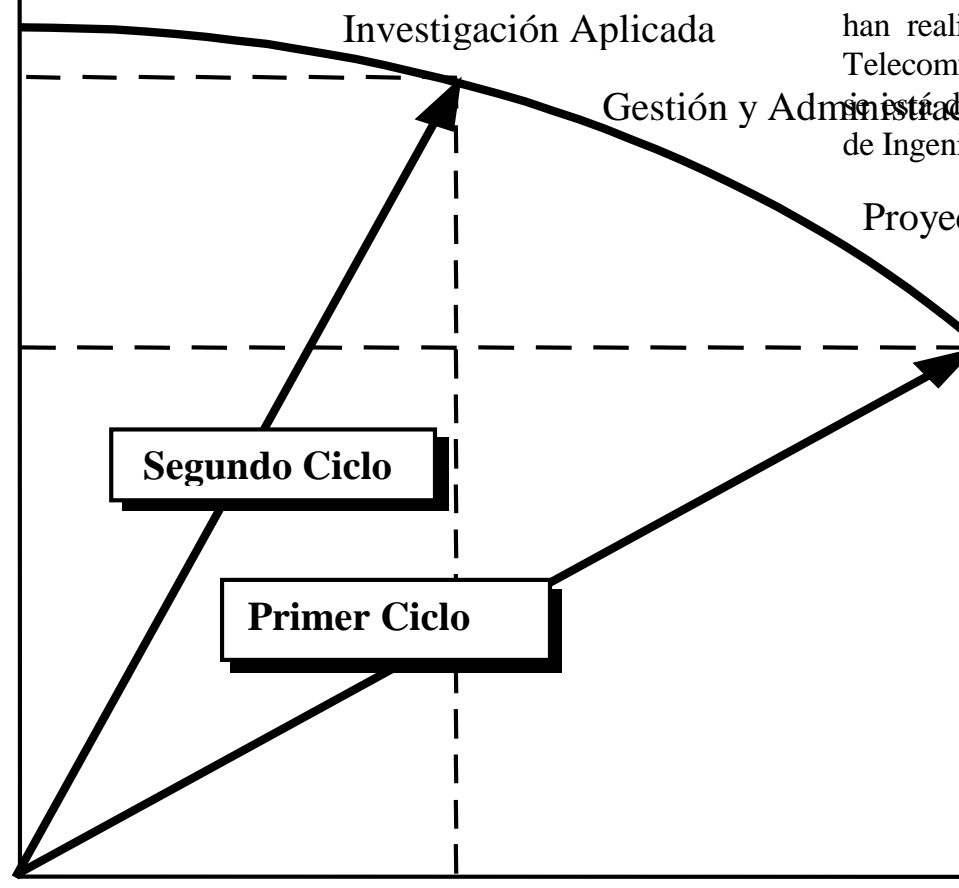

Las dimensiones del diagrama propuesto son:

1. Especialismo/Generalismo

2. Tecnicismo/Humanismo

3. Know-how/Know-why

4. Abstracción/Realismo

5. Producción de Tecnología/Producción de Aplicaciones

El diagrama está dividido en cuatro niveles que representan cualitativamente porcentajes del 25, 50, 75 y

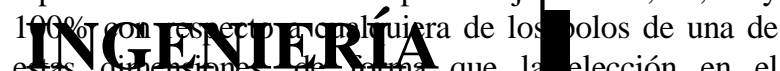
estas aithensibnes, cte rorma que la elección en el

Los Gráficos II-a y II-b muestran las propuestas que se han realizado para los dos grados de la Ingeniería de Telecomunicación y resulta indicativo de la solución que misstŕadión en España al sistema dual en la formación

Proyectos

\section{Producción}

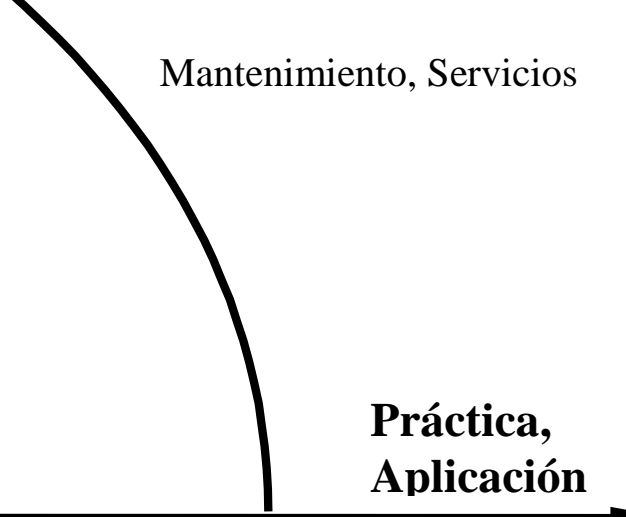

Fig. 3.- Diferente orientación profesional de los dos grados de Ingeniería en España y otros países europeos 


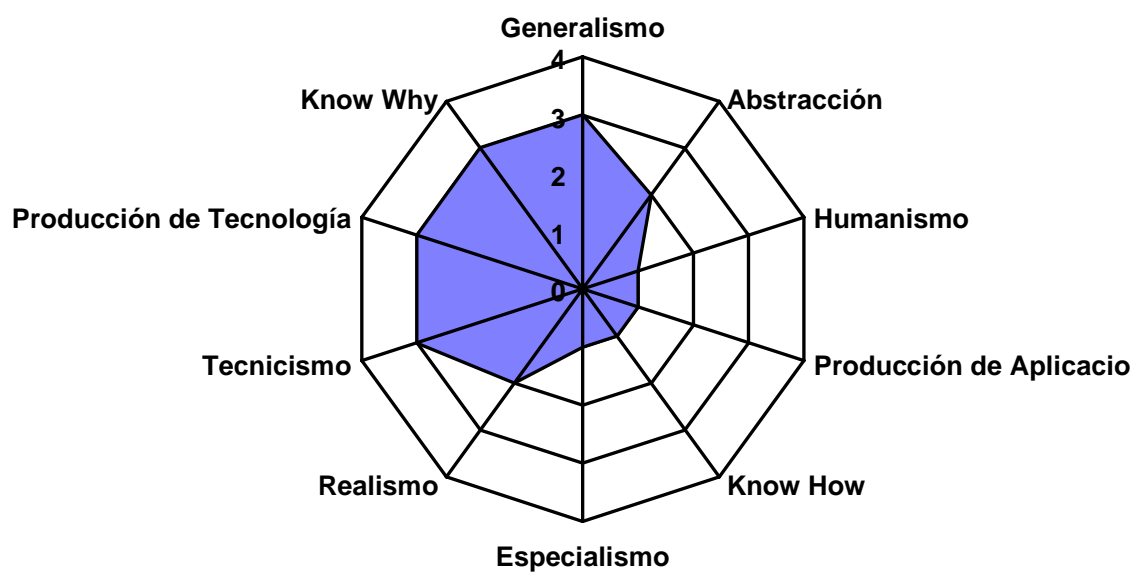

Gráfico II-a.- Propuesta del perfil medio del Ingeniero de Telecomunicación al terminar sus estudios

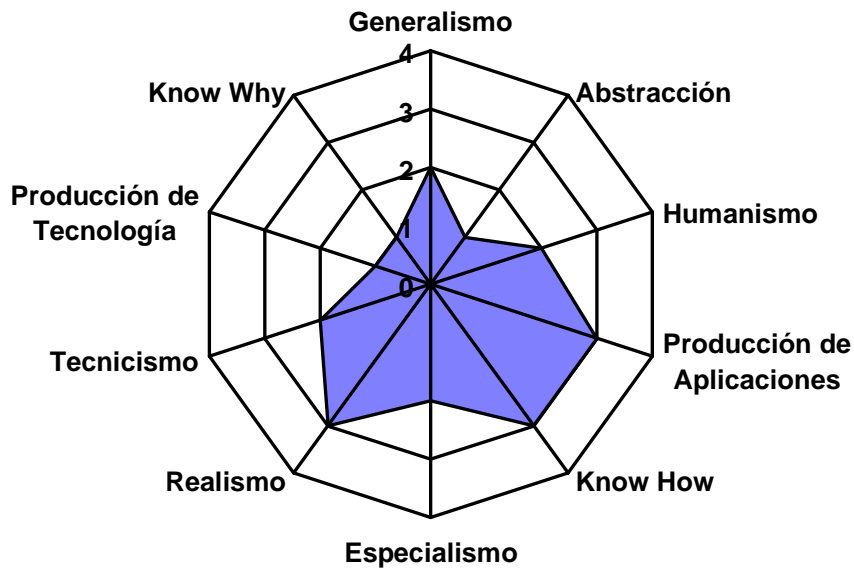

Gráfico II-b.- Propuesta del perfil medio del Ingeniero Técnico de Telecomunicación al terminar sus estudios

La interrelación entre los estudios de primer y segundo ciclo está garantizada por la existencia de pasarelas entre ambas, de forma que el estudiante tiene facilidades para realizar sus estudios de forma cíclica. El paso entre algunos títulos de primer ciclo a otros de segundo ciclo es automático, sin penalización alguna, y está sólo limitado por el número de plazas disponibles en cada Universidad. En otros casos el acceso a segundos ciclos se debe realizar superando unos complementos de formación que dependen en cada caso concreto del curriculum del estudiante. Con esta solución se permite optimizar el esfuerzo del estudiante y rehacer la pirámide educativa de forma que el número de Ingenieros Técnicos sea superior al de Ingenieros de segundo ciclo.

\section{LA UNIVERSIDAD POLITÉCNICA DE MADRID}

La Universidad Politécnica de Madrid (UPM) fue creada en 1971 como tal Universidad, si bien la mayoría de sus centros son más que centenarios pues fueron fundados en los siglos XVIII y XIX y cada uno de ellos mantuvo su vida independiente hasta ser agrupados en la UPM. No es exagerado afirmar que una gran parte de la historia de la tecnología española de más de siglo y medio ha sido escrita por las Escuelas de Arquitectura e Ingenierías de esta Universidad, ya que fueron durante muchísimos años prácticamente las únicas y en algunos casos exclusivamente las únicas, y todas las grandes figuras de la docencia e investigación pasaron por los respectivos 
Centros como alumnos o profesores.

En la actualidad es la Universidad que más proyectos de investigación desarrolla en el área de Ingeniería tanto a nivel nacional como internacional.

\section{CONCLUSIONES}

Desde el punto de vista de la docencia impartida, se pueden agrupar en tres grandes ofertas:

a. Titulaciones impartidas: El proceso de reforma de Planes de Estudio iniciado a raíz de la publicación de la Ley de Reforma Universitaria todavía ha sido completado en algunas de las titulaciones y por tanto conviven todavía algunos planes antiguos con planes renovados. Los centros y titulaciones impartidos por la UPM se muestran en la Tabla IV.

b. Cursos de Postgrado: La Universidad Politécnica de Madrid desarrolla los siguientes tipos de cursos de Postgrado:

- Cursos y seminarios de Formación Continua, de cualquier duración, orientados a la ampliación o actualización de conocimientos siguiendo la evolución de las diversas tecnologías, sus fundamentos y sus aplicaciones.

- Cursos de Especialización, de una duración no inferior a diez créditos, dirigidos a profundizar en temas concretos.

- Cursos de Master o Magister, de una duración no inferior a cincuenta créditos, dirigidos a proporcionar un alto nivel de formación y especialización profesional.

La superación de estos cursos da lugar al correspondiente Título o Certificado acreditativo.

El crecimiento de los estudios de Postgrado desde el año 1988 hasta la fecha ha sido considerable, no sólo en la Universidad Politécnica de Madrid, sino en una gran mayoría de las Universidades avanzadas. En esta Universidad se ha pasado de 11 cursos de Master, 1 Curso de Especialización y 147 de Formación Continua en 1988, a 62 cursos de Master, 154 Cursos de Especialización y 162 de Formación Continua en el año 2000.

Tabla IV.- Centros y Titulaciones de la Universidad Politécnica de Madrid

\begin{tabular}{|c|c|}
\hline CENTROS & TITULACIONES \\
\hline E.T.S ARQUITECTURA & Arquitecto \\
\hline E.T.S.I. AERONÁUTICOS & Ingeniero Aeronáutico \\
\hline E.T.S.I. AGRÓNOMOS & Ingeniero Agrónomo \\
\hline $\begin{array}{l}\text { E.T.S.I. CAMINOS } \\
\text { CANALES Y PUERTOS }\end{array}$ & $\begin{array}{l}\text { Ingeniero de Caminos, } \\
\text { Canales y Puertos }(*)\end{array}$ \\
\hline E.T.S.I. INDUSTRIALES & Ingeniero Industrial (*) \\
\hline E.T.S.I. MINAS & $\begin{array}{l}\text { Ingeniero Geólogo } \\
\text { Ingeniero de Minas }\end{array}$ \\
\hline E.T.S.I. MONTES & Ingeniero de Montes (*) \\
\hline E.T.S.I. NAVALES & Ingeniero Naval $(*)$ \\
\hline $\begin{array}{l}\text { E.T.S.I. } \\
\text { TELECOMUNICACIÓN }\end{array}$ & $\begin{array}{ll}\text { Ingeniero } & \text { de } \\
\text { Telecomunicación } & \\
\end{array}$ \\
\hline $\begin{array}{l}\text { FACULTAD DE } \\
\text { INFORMÁTICA }\end{array}$ & Ingeniero en Informática \\
\hline $\begin{array}{l}\text { E.U. ARQUITECTURA } \\
\text { TÉCNICA }\end{array}$ & to Técnico \\
\hline E.U.I.T. AERONÁUTICA & $\begin{array}{ll}\text { Ingeniero } & \text { Técnico } \\
\text { Aeronáutico }(*) & \\
\end{array}$ \\
\hline E.U.I.T. AGRÍCOLA & $\begin{array}{l}\text { Ingeniero } \\
\text { Agrícola }\end{array}$ \\
\hline E.U.I.T. FORESTAL & $\begin{array}{l}\text { Ingeniero } \\
\text { Forestal } \\
\end{array}$ \\
\hline E.U.I.T. INDUSTRIAL & $\begin{array}{l}\text { Ingeniero } \\
\text { Industrial }\end{array}$ \\
\hline $\begin{array}{l}\text { E.U.I.T. OBRAS } \\
\text { PÚBLICAS }\end{array}$ & $\begin{array}{l}\text { Ingeniero Técnico de } \\
\text { Obras Públicas }\end{array}$ \\
\hline E.U.I.T. TOPOGRÁFICA & $\begin{array}{l}\text { Ingeniero Técnico en } \\
\text { Topografía }\end{array}$ \\
\hline $\begin{array}{l}\text { E.U.I.T. } \\
\text { TELECOMUNICACIÓN }\end{array}$ & $\begin{array}{l}\text { Ingeniero Técnico de } \\
\text { Telecomunicación }\end{array}$ \\
\hline E.U. DE INFORMÁTICA & $\begin{array}{l}\text { Ingeniero Técnico en } \\
\text { Informática }(*)\end{array}$ \\
\hline $\begin{array}{l}\text { ESCUELA } \\
\text { POLITÉCNICA DE } \\
\text { ENSEÑANZA } \\
\text { SUPERIOR }\end{array}$ & $\begin{array}{l}\text { Licenciado en Ciencias } \\
\text { Ambientales } \\
\text { Ingeniero en Geodesia y } \\
\text { Cartografía } \\
\text { Ingeniero de Materiales }\end{array}$ \\
\hline I.N.E.F. & $\begin{array}{l}\text { Licenciado en Ciencias } \\
\text { de la Actividad } \\
\text { Física y del Deporte }\end{array}$ \\
\hline
\end{tabular}

(*) Denominación del título "NO RENOVADO" la mayoría tienen especialidades que figurarán en el reverso de los títulos que se expidan.

El acceso a los Estudios de Postgrado de la Universidad Politécnica de Madrid está regulado por la Normativa de Estudios de Postgrado, que rige todo lo concerniente a 
estos estudios. En principio, será condición necesaria para su inscripción estar en posesión de un título universitario (de Licenciado, Ingeniero, Arquitecto, Diplomado, Ingeniero Técnico o Arquitecto Técnico), quedando a cargo de la dirección del curso la selección de los candidatos de acuerdo con sus conocimientos o su experiencia. El acceso de quienes se hallen en posesión de un título extranjero se realiza sin necesidad de homologación de dicho título (artículo 16.1 del Real Decreto 86/1987 de 16 de enero B.O.E. 23/1/87), bastando la autorización del Rector que la concederá previa comprobación del referido Título y de la documentación académica adicional complementaria presentada, debidamente legalizada, por parte de la Comisión de Postgrado.

c. Doctorado: Los estudios de tercer ciclo en la Universidad Politécnica de Madrid ha sido tradicionalmente uno de los pilares en donde futuros investigadores se han formado científica y metodológicamente. De los diferentes programas de doctorado que imparte la UPM merecen especial interés los desarrollados en Universidades Latinoamericanas .

\section{REFERENCIAS}

[1] Consejo de Universidades. Secretaría General. Guía de la Universidad. Año 1996.

[2] Consejo de Universidades. Secretaría General. La Reforma Universitaria Española: Evaluación e Informe. Febrero 1988.

[3] Ministerio de Educación y Cultura. páginas WEB. http://www.mec.es, 2000.

[4] Gerardo L. Araujo; "Telecommunications Engineering in Spain: A case of curriculum design". Proceedings Frontiers in Education. $22^{\text {nd }}$ Annual Conference.

[5] J. Arriaga; "The three year Engineering Curriculum: A difficult balance". Proceedings Frontiers in Education. $23^{\text {nd }}$ Annual Conference.

[6] J. Arriaga y otros; "EE Education in Spain" IEEE potentials. Feb/march 1999.

[7] S. Vacas, "Reflexiones sobre el modelo educativo universitario de Informática". Aula Fundesco. 1993.

[8] La U.P.M. en Iberoamérica. CDROM editado por la Universidad Politécnica de Madrid. 2000.
[9] Proceedings of III Congreso Nacional de Ingeniería. "Técnica y Sociedad en el umbral del siglo XXI". Madrid. Junio de 1991.

[10] Fédération Européene d'Associations Nationales d'Ingénieurs. "European Engineering Yearbook1994". 